\begin{tabular}{|c|l|}
\hline Title & Direct access to the dispersion relations of multiple ani sotropic surface acoustic modes by Fourier image analysis \\
\hline Author(s) & Sugawara, Y.; Wright, O. B.; Matsuda, O. \\
\hline Citation & $\begin{array}{l}\text { APPLIED PHY SICS LETTERS, 83/7), 1340-1342 } \\
\text { https://doi.org/10.1063/1602151 }\end{array}$ \\
\hline Issue Date & 2003-08-18 \\
\hline Doc URL & http://hdl.handle.net/2115/5790 \\
\hline Rights & Copyright $\odot 2003$ A merican Institute of Physics \\
\hline Type & article \\
\hline File Information & APL83-7.pdf \\
\hline
\end{tabular}

Instructions for use 


\title{
Direct access to the dispersion relations of multiple anisotropic surface acoustic modes by Fourier image analysis
}

\author{
Y. Sugawara, O. B. Wright, ${ }^{\text {a) }}$ and O. Matsuda \\ Department of Applied Physics, Faculty of Engineering, Hokkaido University, Sapporo 060-8628, Japan
}

(Received 14 April 2003; accepted 17 June 2003)

\begin{abstract}
We present a method based on a combination of temporal and spatial Fourier image analysis, involving the incorporation of a time-reversed acoustic propagation component, that can be used to obtain the angular dispersion or general dispersion relations of multiple surface acoustic modes excited by a localized pulsed source. The method is applied to the study of acoustic propagation at the surface of the highly anisotropic single-crystal $\mathrm{TeO}_{2}$ coated with a thin gold film. (C) 2003 American Institute of Physics. [DOI: 10.1063/1.1602151]
\end{abstract}

Surface acoustic wave propagation on bulk materials and thin film structures has proved of fundamental and practical interest for studying anisotropy and measuring film thicknesses or elastic properties. ${ }^{1-6}$ A common method for such investigations is to use plane acoustic wave fronts, and to repeat the experiment for each propagation direction sequentially. However, the imaging of acoustic waves emanating from a point source is very useful for materials characterization because phenomena dependent on propagation direction, such as surface phonon focusing, can be effectively and immediately recorded with an omnidirectional acoustic wave vector distribution. ${ }^{7-11}$ For surface acoustic imaging with impulsive point sources, the group velocity is readily measured from the wave front shape. However, it is the phase velocity-or its reciprocal, the slowness-that is more directly related to material elastic properties. ${ }^{12}$ Impulsive point-source acoustic excitation of anisotropic samples by nature involves the generation of several surface and bulk acoustic modes that can produce complex overlapping wave fronts; no general method for the direct and automatic extraction of the individual dispersion relations (and hence the phase velocities) from such experimental images involving multiple propagating modes traveling parallel to the surface has been proposed. Previous methods can only be applied to images where only one acoustic mode propagates in any particular direction. ${ }^{9}$ In this letter we present a general technique for analysis in time-resolved acoustic imaging at surfaces in the two lateral spatial dimensions that allows the extraction of the full set of dispersion relations for all excited modes simultaneously.

We demonstrate the method with experiments on the transparent tetragonal crystal $\mathrm{TeO}_{2}$ cut in the (001) orientation and coated with a thin polycrystalline gold film of thickness $(40 \mathrm{~nm})$ small enough to avoid significant thin film dispersion here and thus simplify comparison with theory. This crystal is strongly anisotropic in this cut and results in a complex phonon focusing pattern, ideal for evaluating techniques for acoustic mode analysis. ${ }^{9,13}$ We use an ultrafast optical pump and probe technique based on a common-path interferometer. ${ }^{14}$ Acoustic waves are thermoelastically ex-

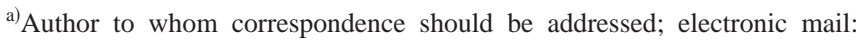
assp@kino-ap.eng.hokudai.ac.jp
}

cited at a $\sim 4 \mu \mathrm{m}$ Gaussian spot (full width at half maximum intensity) in the sample using optical pulses normally incident from the substrate side at wavelength $415 \mathrm{~nm}$, repetition rate $80 \mathrm{MHz}$, duration $\sim 1 \mathrm{ps}$, and optical fluence $\sim 2$ $\mathrm{mJ} \mathrm{cm}{ }^{-2}$, producing an initial transient temperature change $\sim 200 \mathrm{~K}$. With this impulsive source, broadband acoustic excitation up to $\sim 1 \mathrm{GHz}$ is achieved in the lateral direction, with maximum efficiency in the $300-700 \mathrm{MHz}$ range (or wavelength $\sim 7 \mu \mathrm{m}$ ). Out-of-plane surface motion (along the $z$ axis) is detected interferometrically with $\sim 0.4$ pm resolution by the use of two optical probe pulses of wavelength $\lambda=830 \mathrm{~nm}$ at an interval of $\tau=330 \mathrm{ps}$, focused at normal incidence to the same spot size on the front surface of the film. The output beam from the interferometer is divided into two using a polarizing beam splitter. These beams are fed to two photodetectors, allowing the optical phase difference $\Delta \phi$ of the two probe pulses to be obtained. The relative position of the pump and probe spots is scanned for a selection of pump-probe delay times, building up a full animation of the surface acoustic propagation over the interval $T=12.5 \mathrm{~ns}$ between optical pump pulses. For the gold film and probe wavelength used, $\Delta \phi$ is only sensitive to the out-of-plane surface motion. ${ }^{14}$ We therefore express our data in terms of the calibrated difference in surface displacement $\Delta u_{z}$ $=\lambda \Delta \phi / 4 \pi$ recorded by the two probe pulses (that is effectively averaged over the probe beam spatial profile). This difference $\Delta u_{z}$ is proportional to the outward surface particle velocity $v_{z}=\partial u_{z} / \partial t$, where $v_{z} \approx \Delta u_{z} / \tau$ (since $\tau \ll 2 \pi / \omega$, where $\omega$ is the phonon angular frequency).

An animation consisting of 23 images in a series separated by the time interval 533 ps was obtained for a $60 \mu \mathrm{m}$ $\times 60 \mu \mathrm{m}$ region $(120 \times 120$ pixels $)$ of the surface. The lateral resolution is, however, limited to $\sim 4 \mu \mathrm{m}$ by the probe beam diameter. Figures 1(a) and 1(b) show two such images of $\Delta u_{z}$ for delay times 4.0 and $8.5 \mathrm{~ns}$ after the pump excitation (at the image center). Multiple wave fronts are produced by expanding wavepackets according to the 12.5 ns pulse interval. These are shaped as the group velocity surfaces, which have fourfold symmetry. ${ }^{9,13}$ Such a time series of frames of the acoustic propagation intuitively can be seen to contain sufficient information for the extraction of the acoustic dispersion relations. The object is to achieve this in a simple 


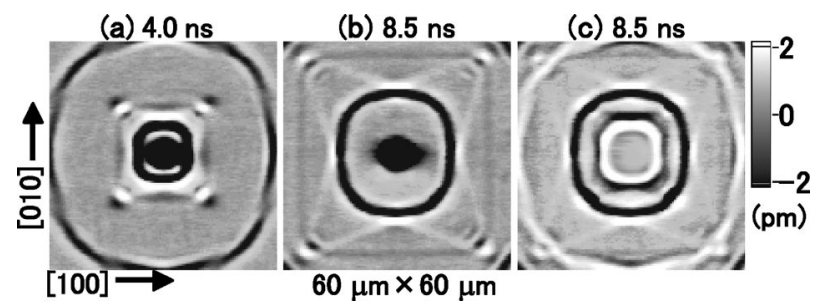

FIG. 1. (a)-(b) Experimental images of surface acoustic propagation on a $60 \mu \mathrm{m} \times 60 \mu \mathrm{m}$ region of $(001) \mathrm{TeO}_{2}$ of thickness $1 \mathrm{~mm}$ coated with a 40 $\mathrm{nm}$ polycrystalline gold film for the fixed delay times 4.0 and $8.5 \mathrm{~ns}(=-4.0$ ns), respectively. (c) An example of a processed image that includes an inverted time-reversed propagating component. This image, for delay time $8.5 \mathrm{~ns}$, corresponds to the difference of the images in (a) and (b). The white color represents the outward direction for $v_{z}$. The gray scale has been truncated at $\Delta u_{z}= \pm 2 \mathrm{pm}$ for clarity, but amplitudes up to $\sim \pm 8 \mathrm{pm}$ are in fact present in the propagating portions of these images.

and automatic way, avoiding the need for time-consuming recursive optimization routines.

We propose such a method based on a dual Fourier transformation, first taking a two-dimensional spatial Fourier transform $F(\mathbf{k}, t)$ of the acoustic disturbance $f(\mathbf{r}, t)$ $=\Delta u_{z}(\mathbf{r}, t):{ }^{15}$

$$
\begin{aligned}
F(\mathbf{k}, t)= & \frac{1}{(2 \pi)^{2}} \int_{-\infty}^{\infty} f(\mathbf{r}, t) \exp (-i \mathbf{k} \cdot \mathbf{r}) d^{2} \mathbf{r} \\
= & \frac{1}{2} \sum_{n}\left\{F_{n}(\mathbf{k}) \exp \left[-i \omega_{n}(\mathbf{k}) t\right]\right. \\
& \left.+F_{n}^{*}(-\mathbf{k}) \exp \left[i \omega_{n}(\mathbf{k}) t\right]\right\},
\end{aligned}
$$

where

$$
\begin{aligned}
f(\mathbf{r}, t) & =\int_{-\infty}^{\infty} F(\mathbf{k}, t) \exp [i \mathbf{k} \cdot \mathbf{r}] d^{2} \mathbf{k} \\
& =\operatorname{Re} \sum_{n} \int_{-\infty}^{\infty} F_{n}(\mathbf{k}) \exp \left[i\left(\mathbf{k} \cdot \mathbf{r}-\omega_{n}(\mathbf{k}) t\right)\right] d^{2} \mathbf{k}
\end{aligned}
$$

Here $\mathbf{k}$ is the wave vector, $n$ is an index labeling the acoustic modes, and $\omega=\omega_{n}(\mathbf{k})$ is the dispersion relation. We have assumed $\omega_{n}(\mathbf{k})=\omega_{n}(-\mathbf{k})$ because the sample is laterally homogeneous, and, for simplicity, have neglected the effects of acoustic attenuation $\left[\omega_{n}(\mathbf{k})=\omega_{n}^{*}(\mathbf{k})\right]$. The functions $F_{n}(\mathbf{k})$ represent the spectral content of each acoustic mode $n$. We take a second Fourier transform in the temporal domain

$$
\begin{aligned}
F(\mathbf{k}, \omega)= & \frac{1}{2 \pi} \int_{-\infty}^{\infty} F(\mathbf{k}, t) \exp (i \omega t) d t \\
= & \frac{1}{4 \pi} \sum_{n}\left(F_{n}(\mathbf{k}) \int_{-\infty}^{\infty} \exp \left\{i\left[\omega-\omega_{n}(\mathbf{k})\right] t\right\} d t\right. \\
& \left.+F_{n}^{*}(-\mathbf{k}) \int_{-\infty}^{\infty} \exp \left\{i\left[\omega+\omega_{n}(\mathbf{k})\right] t\right\} d t\right) .
\end{aligned}
$$

Equation (3) can be converted to the simple form

$$
\begin{aligned}
F(\mathbf{k}, \omega)= & \frac{1}{2} \sum_{n} F_{n}(\mathbf{k}) \delta\left[\omega-\omega_{n}(\mathbf{k})\right] \\
& +F_{n}^{*}(-\mathbf{k}) \delta\left[\omega+\omega_{n}(\mathbf{k})\right],
\end{aligned}
$$
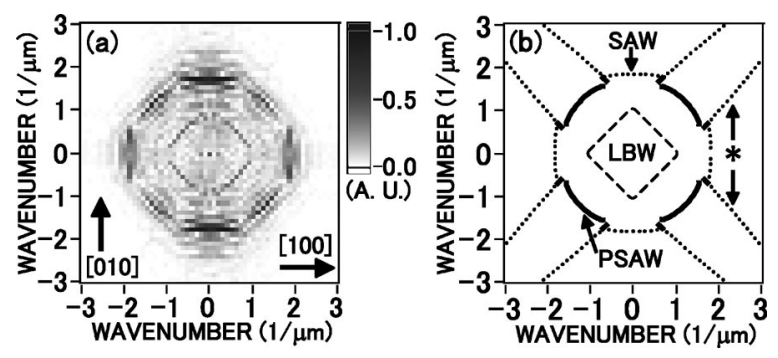

FIG. 2. (a) Constant frequency surface of the experimentally-determined function $|G(\mathbf{k}, \omega)|$ for $\omega / 2 \pi=570 \mathrm{MHz}$. (b) The result of theoretical calculations of the slowness surfaces for SAW, PSAW, and LBW represented with the horizontal scales $k_{x}$ and $k_{y}$ instead of the usual $k_{x} / \omega$ and $k_{y} / \omega$, for $\omega / 2 \pi=570 \mathrm{MHz}$. The star represents SAW branches not observed in experiment.

closely related to the dispersion relation, being only nonzero for $(\omega, \mathbf{k})$ satisfying $\omega= \pm \omega_{n}(\mathbf{k})$.

Equations (1)-(4) strictly apply only when the waves have been propagating for an infinite time with no acoustic attenuation; there should be no acoustic sources present to contribute to the acoustic disturbance. In our case the surface acoustic waves are generated in a localized region of the sample and propagate outwards. In order to convert our experimental data into a form compatible with the earlier theory, we add to $f(\mathbf{r}, t)$ an inverted time-reversed component $-f(\mathbf{r},-t)$ representing incoming wave fronts, matching the two sets of data to produce a combination of converging and diverging acoustic waves, with no acoustic sources. The need for the sign inversion of $f$ stems from imaging the outward surface velocity $v_{z}\left(\propto \Delta u_{z}\right)$ rather than the surface displacement $u_{z} \cdot{ }^{16}$ We therefore choose $g(\mathbf{r}, t)=f(\mathbf{r}, t)$ $-f(\mathbf{r},-t)$ and its dual Fourier transform $|G(\mathbf{k}, \omega)|$ for image analysis. The time $t=0$, for which $g(\mathbf{r}, 0)=0$, corresponds to the moment an incoming wave front is matched to an outgoing one. Figure 1(c) shows an example of a processed image corresponding to $g(\mathbf{r}, t)$, in which a window function has been used to remove the central spot associated with thermal expansion and temperature changes in the acoustic generation zone. ${ }^{17}$ This method, based on the incorporation of a time-reversed acoustic field, shows interesting parallels with the more active methods of acoustic time-reversal. ${ }^{18}$

To obtain the dispersion relations from the experiment, we evaluate $|G(\mathbf{k}, \omega)|$ using the numerical Fourier transforms of the processed time-resolved data for $\mathrm{TeO}_{2} \cdot{ }^{19}$ Figure 2(a) shows an example of a section of the experimentally determined function $|G(\mathbf{k}, \omega)|$ for $\omega / 2 \pi=570 \mathrm{MHz}$. This corresponds to a constant frequency surface in wave vector space. Figure 2(b) shows the corresponding result of theoretical calculations for surface acoustic waves (SAW), pseudosurface acoustic waves (PSAW) and quasi-longitudinal bulk waves (LBW) (traveling parallel to the surface) on the same scale, ${ }^{1,13,20}$ that is the slowness surfaces for these modes with the conventional axes $k_{x} / \omega$ and $k_{y} / \omega$ multiplied by the constant value of $\omega$ to give $k_{x}$ and $k_{y} .{ }^{21}$ (We have ignored small film loading and piezoelectric effects in the calculation.) The lines traced out by the experimental $|G(\mathbf{k}, \omega)|$ are reproduced well by the theory. However, the combined effects of phonon focusing and the selective detection of the out-ofplane motion prevent SAW branches of the type marked with 


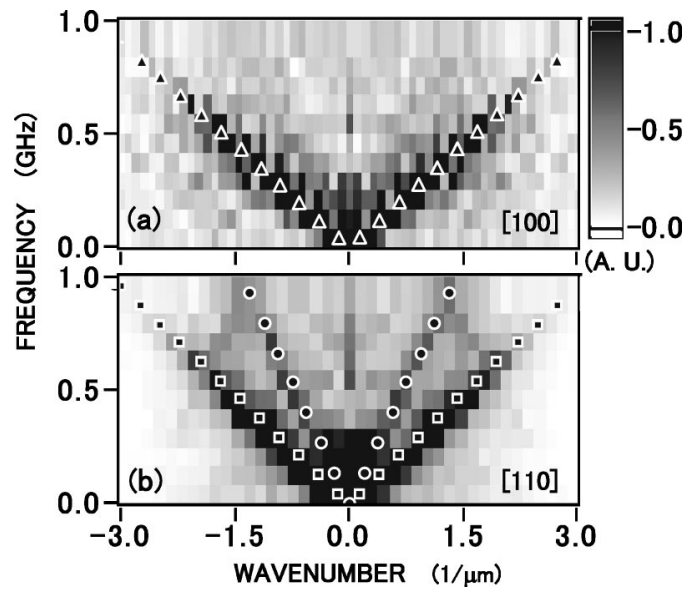

FIG. 3. Dispersion relations (cross sections of the experimentally determined function $|G(\mathbf{k}, \omega)|$ ) for the directions (a) [100] and (b) [110], respectively, together with theoretical calculations for SAW (triangles), PSAW (squares), and LBW propagating parallel to the surface (circles).

the star in Fig. 2(b) being detected. ${ }^{13}$ The square feature in the center of Fig. 2(a) arises from a LBW traveling in the near-surface region. ${ }^{22}$ The deviations from fourfold symmetry observed in $|G(\mathbf{k}, \omega)|$ are probably caused by residual ellipticity of the optical pump spot. [The image analysis we have adopted implies $|G(\mathbf{k}, \omega)|=|G(-\mathbf{k}, \omega)|$.]

The equivalence of $|G(\mathbf{k}, \omega)|$ and the dispersion relation is even more evident in Figs. 3(a) and 3(b), that show $\omega / 2 \pi$ vs $k$ obtained from sections of the experimental $|G(\mathbf{k}, \omega)|$ for the fixed propagation directions [100] and [110], respectively. Shown superimposed are the theoretical calculations of $\omega / 2 \pi$ versus $k$ for SAW (triangles), PSAW (squares), and LBW (circles). As expected for a bulk sample, for each direction the phase velocities are independent of $k$. (For [100] $\omega / k \approx 1.89 \mathrm{~km} \mathrm{~s}^{-1}$ for SAW. For [110], $\omega / k \approx 2.01 \mathrm{~km} \mathrm{~s}^{-1}$ for PSAW, and $4.47 \mathrm{~km} \mathrm{~s}^{-1}$ for LBW.) Good agreement is obtained for those regions of $\omega-\mathbf{k}$ space that are significantly excited.

An interesting experimental limitation concerns the imaged area $L \times L$. Fourier analysis of this finite region leads to a minimum resolvable step in $\mathbf{k}$ equal to $2 \pi / L\left(\approx 0.1 \mu \mathrm{m}^{-1}\right.$ for $L=60 \mu \mathrm{m}$ ) and to the appearance of small oscillations surrounding the maxima of $|G(\mathbf{k}, \omega)|$. These effects, rather than the neglect of acoustic attenuation (which has a negligible influence for our image size) ${ }^{20}$ are the dominant sources of error in the data analysis. The periodic laser excitation also restricts the spectrum $|G(\mathbf{k}, \omega)|$ to values of $\omega / 2 \pi$ that are integer multiples of the $80 \mathrm{MHz}$ repetition rate. However, this does not pose problems in the analysis or in the evaluation of the crystal for the present measurements.

In conclusion, we have presented a method that can be used to directly obtain the dispersion relations of multiple surface acoustic modes from time-resolved two-dimensional images of anisotropic samples. It allows the immediate conversion of complex experimental wave fronts to slowness surfaces convenient for comparison with theoretical models. Our experiments involve an impulsive and approximately axially symmetric acoustic source, but the analysis is general, and could be applied to other temporal wave forms or spatial source shapes. Although the film in the present study did not significantly affect the acoustic dispersion over the region probed, for thicker films the method could be used for the nondestructive measurement of film thickness. We envisage application to wide range of crystals as well as to substrates with texture on micron or nanometer scales.

The authors are indebted to S. Tamura, Y. Tanaka, and V. E. Gusev for valuable discussions on the theory of acoustic dispersion.

${ }^{1}$ G. W. Farnell and E. L. Adler, in Physical Acoustics, edited by W. P. Mason and R. N. Thurston (Academic, New York, 1972), Vol. 9, p. 35.

${ }^{2}$ J. Rogers, A. A. Maznev, M. J. Banet, and K. A. Nelson, Annu. Rev. Mater. Sci. 30, 117 (2000).

${ }^{3}$ C. Glorieux, W. Gao, S. E. Cruger, K. Van de Rostyne, W. Lauriks, and J. Thoen, J. Appl. Phys. 88, 4394 (2000).

${ }^{4}$ M. Szabadi, P. Hess, A. J. Kellock, H. Coufal, and J. E. E. Bablin, Phys. Rev. B 58, 8941 (1998).

${ }^{5}$ D. Shilo and E. Zolotoyabko, Ultrasonics 40, 921 (2002).

${ }^{6}$ D. Xiang, N. N. Hsu, and G. V. Blessing, Appl. Phys. Lett. 74, 2236 (1999).

${ }^{7}$ R. E. Vines, S. Tamura, and J. P. Wolfe, Phys. Rev. Lett. 74, 2729 (1995).

${ }^{8}$ M. Pluta, A. G. Every, W. Grill, and T. J. Kim, Phys. Rev. B 67, 094117 (2003).

${ }^{9}$ Y. Sugawara, O. B. Wright, O. Matsuda, M. Takigahira, Y. Tanaka, S. Tamura, and V. E. Gusev, Phys. Rev. Lett. 88, 185504 (2002).

${ }^{10}$ Y. Sugawara, O. B. Wright, and O. Matsuda, Rev. Sci. Instrum. 74, 519 (2003).

${ }^{11}$ K. L. Telschow, V. A. Deason, R. S. Schley, and S. M. Watson, J. Acoust. Soc. Am. 106, 2578 (1999).

${ }^{12}$ A. G. Every and W. Sachse, Phys. Rev. B 42, 8196 (1990).

${ }^{13}$ Y. Tanaka, M. Takigahira, and S. Tamura, Phys. Rev. B 66, 075409 (2002).

${ }^{14}$ D. H. Hurley and O. B. Wright, Opt. Lett. 24, 1305 (1999).

${ }^{15} \mathrm{We}$ select here the $1 / 2 \pi$ factor for forward Fourier transforms.

${ }^{16}$ Our analysis is based on observations in Y. Sugawara, O. B. Wright, O. Matsuda, M. Takigahira, Y. Tanaka, S. Tamura, and V. E. Gusev, Phys. Rev. Lett. 88, 185504 (2002) for isotropic substrates suggesting that $F_{n}(\mathbf{k})$ is imaginary (for $v_{z}$ images). A similar analysis for $u_{z}$ images would therefore require real $F_{n}(\mathbf{k})$ and the use of $u_{z}(\mathbf{r}, t)+u_{z}(\mathbf{r},-t)$ instead of $f(\mathbf{r}, t)-f(\mathbf{r},-t)$. Trial use of $f(\mathbf{r}, t)+f(\mathbf{r},-t)$ lead to broadened spectral features.

${ }^{17}$ The window function is $w(x, y)=1-\exp \left[-\left(x^{2}+y^{2}\right)^{2} / 50^{2}\right]$, with $x$ and $y$ in microns.

${ }^{18}$ J. de Rosny and M. Fink, Phys. Rev. Lett. 89, 219901 (2002).

${ }^{19}$ To avoid temporal truncation effects, the ideal integration range for the temporal Fourier transform is over $T=12.5 \mathrm{~ns}$. We checked that the chosen range of $12.26 \mathrm{~ns}$ leads to a negligible shift in the maxima of $|G(\mathbf{k}, \omega)|$ for a given $\omega$. Images of $|F(\mathbf{k}, \omega)|$, calculated for comparison, show a degraded spectrum with broadened peaks at $\omega=\omega_{n}(\mathbf{k})$.

${ }^{20}$ B. A. Auld, Acoustic Fields and Waves in Solids (Wiley, New York, 1973), Vols. I and II.

${ }^{21}$ The elastic constants for $\mathrm{TeO}_{2}$ are taken as $c_{11}=55.7, c_{12}=51.2, c_{13}$ $=21.8, c_{33}=105.8, c_{44}=26.5$, and $c_{66}=65.9$ (in units of gigapascal), with density $\rho=5.99 \mathrm{~g} \mathrm{~cm}^{-3}$.

${ }^{22}$ The bulk quasitransverse mode, that becomes pure longitudinal in the [100] or [010] directions [see B. A. Auld, Acoustic Fields and Waves in Solids (Wiley, New York, 1973), Vol. I], appears not to be significantly excited. In addition, the LBW mode becomes pure shear horizontal in these directions, and by symmetry is not excited. 
Applied Physics Letters is copyrighted by the American Institute of Physics (AIP). Redistribution of journal material is subject to the AIP online journal license and/or AIP copyright. For more information, see http:/ojps.aip.org/aplo/aplcr.jsp

Copyright of Applied Physics Letters is the property of American Institute of Physics and its content may not be copied or emailed to multiple sites or posted to a listserv without the copyright holder's express written permission. However, users may print, download, or email articles for individual use. 